\title{
Nuevos contenidos y nuevos métodos en Narrativa Audiovisual
}

\author{
Pedro Javier GóMEZ MARTíNEZ \\ Universidad Francisco de Vitoria \\ p.gomez.prof@ufv.es \\ Miguel Ángel Poveda CRIADO \\ Universidad Francisco de Vitoria \\ m.poveda.prof@ufv.es
}

Recibido: $10 / 10 / 2012$

Aceptado: 23/01/2013

\section{Resumen}

El presente artículo muestra los primeros resultados de un proyecto de innovación docente financiado por la Universidad Francisco de Vitoria, de Madrid, orientado a conocer las necesidades formativas del personal cualificado que se dedica a las tareas de creación de contenidos en el ámbito de la ficción, así como a analizar la oferta académica de las universidades españolas en este sentido, para determinar en qué medida, lo que se enseña y la metodología que se emplea en la forma de impartirlo, responde a las necesidades del escenario actual. El objetivo de la investigación no es otro que el de adecuar la formación de los estudiantes de las titulaciones del Grado en Comunicación en las áreas relacionadas con la Narrativa, a las exigencias de los momentos actuales y de los inmediatamente venideros.

Palabras clave: Narrativa Audiovisual, Contenidos, Innovación Docente, EEES.

\section{New Contents and New Methods in Audiovisual Narrative}

\begin{abstract}
This article shows the first results of an educational innovation project funded by the Universidad Francisco de Vitoria of Madrid, oriented to meet the training needs of qualified staff that is dedicated to the task of creating content in the field of fiction, as well as to analyze Spanish universities in this regard academic offer to determine if what is been teaching and the methodology give an answer to the needs of the current scenario. The objective of the research is to adapt the training of the students of the communication degrees in the areas related to the narrative, to the demands of the present time and the immediately coming.
\end{abstract}

Keywords: Audiovisual narrative, content, teaching innovation, ESG.

\section{Referencia normalizada}

GÓMEZ MARTÍNEZ, Pedro Javier y POVEDA CRIADO, Miguel Ángel (2013): "Nuevos contenidos y nuevos métodos en Narrativa Audiovisual". Estudios sobre el Mensaje Periodístico. Vol. 19, Núm. especial marzo, págs.: 223-232. Madrid, Servicio de Publicaciones de la Universidad Complutense.

Sumario: 1. Introducción. 2. Metodología. 3 Desarrollo: la oferta académica, sus carencias y sus nuevos retos; 3.1. Contenidos impartidos u ofertados; 3.2. Metodologías empleadas en las asignaturas; 3.3. Fuentes empleadas en el ejercicio docente. 4. Conclusiones. 5. Referencias bibliográficas.

\section{Introducción}

Se viene observando desde los últimos años, una mayor presencia de nuevos canales, ventanas de distribución y soportes, cuya aparición parece haber incidido considerablemente no sólo en las técnicas y procesos en la elaboración de guiones, sino también en la estructura de los equipos y en las dinámicas de trabajo (López Quintana, 2007: 397), lo que aconseja como mínimo, una revisión de los materiales educativos y de las metodologías docentes. 
La industria pocas veces ha manifestado de una manera explícita su interés por los profesionales más directamente implicados en la elaboración de los contenidos. Tan es así que hasta hace relativamente poco, los guionistas se formaban directamente en su contacto con la profesión. Fue así en el cine, donde prosperaron nombres de la talla de Rafael Azcona, sin más escuela que su propia trayectoria y con una formación teórica, si es que la había, heredada del teatro. El resto les venía como resultado de sus propios errores.

En los años ochenta, algunas voces críticas empezaron a percibir la necesidad de una cualificación exigente para este perfil y comenzaron a publicarse en nuestro país abundantes textos que contribuían a paliar el déficit formativo desde el ámbito universitario. Con ello también, a prestigiar la figura profesional de los guionistas:

"Gracias a esta tendencia ya contamos con una apreciable cantidad de guiones editados, una bibliografía bastante extensa y traducida en estos últimos años, revistas especializadas y una incipiente investigación que empieza a estudiar el guión con la seriedad que le corresponde” (Ríos Carratalá, 2006:12).

Pero hasta la teleficción de los noventa, puede decirse que el panorama no cambió demasiado porque ni siquiera en los planes de estudios de las carreras de comunicación (entonces Imagen y Sonido) se le dedicaba demasiado espacio a la formación estrictamente profesional. Era una preparación tal vez más orientada al perfil del crítico, del especialista académico, si se prefiere, pero decididamente escasa en soluciones para un guionista que debiera enfrentarse al ejercicio de la profesión.

De algún modo se daba por hecho que méritos tales como la originalidad o el buen estilo, siempre muy apreciables en un guión, eran aspectos que no podían enseñarse, ni aprenderse. A lo sumo se llegaba a admitir la existencia de una técnica (propia de cada medio: cine, tv, radio...) cuya enseñanza prácticamente se resolvía en una o dos sesiones en las que se contaba cómo era un guión o qué tipo de información debía aparecer en él. A esto se le podía sumar como mucho algún taller práctico.

Como mencionaba Mckee, una de las principales razones por las que se acumulan toneladas de ideas descartadas en los registros y en las mesas de recepción de proyectos de los grandes estudios, es que ante las buenas ideas se suele ceder a la tentación de intentar la escritura, mucho antes de haber adquirido la pericia necesaria para hacerlo con alguna habilidad.

"The Writers Guild of America script registration services logs over thirty-five thousand titles yearly. These are only those that are registered. Across America hundred of thousands of screenplays are attemped each year, but only a handful are quality screenplays, for many reasons but this above all: Today's would be writers rush to the typewriter without first learning their craft"1 (Mckee, 2002: 32)

1 "El Sindicato de Guionistas de América inscribe en sus registros más de treinta y cinco mil títulos al año. Y ésos son solamente los que se llegan a registrar. En toda América se intentan escribir cientos de miles de guiones cada año, pero sólo hay unos pocos de calidad debido a múltiples razones. La principal es que los que hoy aspiran a ser guionistas se apresuran a sentarse ante el teclado sin aprender primero el oficio". 
Hoy resulta discutible que estas técnicas no puedan enseñarse y aprenderse, constituyendo en sí mismas un importantísimo legado para los proyectos formativos orientados a la innovación y la mejora, a la vez que también un valioso objeto de estudio sobre el que se puede ampliar el conocimiento.

Autores diversos, como Robert y Michèle Root-Berstein se basan, por ejemplo, en "informes introspectivos realizados por los mismos pensadores, creadores e inventores" (Root-Berstein, 2002:17) para conocer a fondo procesos mentales tan abstractos como los que conciernen a la creatividad, con la sola idea de facilitar la posibilidad de entrenarla o mejorarla.

El aprendiz de guionista echaba en falta una mayor atención a los elementos relacionados con la escritura y la dramaturgia del texto de ficción audiovisual, antes del rediseño que los planes de estudios de las carreras de comunicación tuvieron allá por los noventa. Naturalmente se llegó un poco tarde a cubrir tal necesidad, en parte porque se tardó también un cierto tiempo en tomar conciencia de ella.

Los cambios recientes demandan necesidades formativas en los estudiantes de comunicación, que les permitan afrontar mejor los retos del presente. Una de esas necesidades tiene mucho que ver por ejemplo con los contenidos transmedia, un concepto difuso aún, al que nos referimos unas veces para aludir a micro-ficciones derivadas de las series televisivas y en otras ocasiones, a series específicas del medio web y/o las que encuentran difusión por diversos canales (Jenkins, 2006: 81).

¿La aparición de nuevos medios requiere del hallazgo de nuevas fórmulas susceptibles de enseñarse y aprenderse o seguiremos enseñando, como única referencia, el discurso cinematográfico en tanto que base y fundamento de todo lo audiovisual? Hace ya bastante tiempo que esta cuestión quedó resuelta y hoy en la mayor parte de los centros superiores de formación, universitarios o no, se atiende a la especificidad de cada medio.

Las entrevistas con los guionistas nos permitieron comprobar que aún sigue existiendo un margen bastante amplio para el autodidactismo, sólo que ya sobre la base de unos conocimientos ampliamente consensuados, referidos a delimitar qué es lo esencial en cualquier guión (aspectos tales como trucos o consejos para lograr la identificación del espectador, el uso del humor como desengrasante entre momentos trágicos de la historia, etc...). La mayor parte de ellos rechazaba la formación específica y abogaba más por un modelo de búsqueda personal a través de fuentes cuya solvencia viniese abalada por la propia trayectoria profesional de sus autores. Y sin embargo casi todos coincidían en que cada día más, el trabajo del guionista está dejando de ser una actividad individual para convertirse en una labor de equipo, altamente específica y cualificada.

\section{Metodología}

La investigación se realiza sobre una encuesta que recoge un análisis profundo y objetivo de la totalidad de los contenidos de los centros que imparten estas disciplinas en nuestro país, así como de las orientaciones de un panel de expertos, guionistas profesionales y especialistas en el ámbito académico, obtenido después de un encuentro que tuvo lugar el 23 de abril de 2012 en la Universidad Francisco de Vitoria de Ma- 
drid. De manera complementaria se han comparado los contenidos de las diferentes bibliografías recomendadas en las guías docentes publicadas, así como los textos audiovisuales propuestos para el análisis del alumnado.

\section{Desarrollo: la oferta académica, sus carencias y sus nuevos retos}

Como consecuencia de la nueva universidad que en nuestro país trata de implantar el denominado Plan Bolonia, contábamos con una fuente de información bastante fiable, al menos en lo que respecta a las intenciones de cada centro: las denominadas Guías Docentes de las asignaturas, cuya publicación se recomienda. A través de ellas intentamos acceder al retrato robot de lo que se está ofreciendo. De un total de cuarenta y cinco centros superiores universitarios reconocidos oficialmente, se analizaron veintitrés, lo que viene a suponer más de la mitad (el 51.11\%).

¿Por qué no todos? En numerosos casos sencillamente porque no fue posible, no se pudo acceder a esta información debido a motivos muy diversos, entre los que podemos destacar: la guía docente no estaba disponible, la asignatura aún no estaba implementada, lo que se denominaba "guía docente" en realidad no lo era o, simplemente, no nos daba la información buscada. En general, bastaba advertir que la información ofertada resultaba insuficiente, para que de manera inmediata la apartáramos del estudio, con vistas sobre todo a evitar sesgos y errores en los porcentajes.

¿Qué buscábamos en estos documentos? Básicamente tres cosas: contenidos, metodologías y fuentes. Intentábamos saber qué se estaba enseñando, cómo se enseñaba y partiendo de qué corpus teórico. Después de una primera prospección se elaboró un listado amplio de contenidos, en el que se incluyó la totalidad de lo encontrado, para posteriormente ir comparando una a una, la oferta de cada universidad o centro y de este modo ir analizando a qué se le daba prioridad en cada plan de estudios y, así mismo, qué resultaba marginado.

\subsection{Contenidos impartidos u ofertados}

Deseábamos saber si la actual formación estaba abogando por un modelo basado en la práctica de aspectos concretos de la escritura o si, por el contrario, se utilizaba el epígrafe de la materia para insertar conocimientos más generales del medio audiovisual.

Lo primero nos habla de una mayor preocupación en los aspectos profesionalizantes de la materia, con el peligro de que la aplicación práctica le reste peso a las grandes cuestiones de trasfondo humano, filosófico y moral, que son en definitiva la causa y el fin último que se presupone detrás de cada discurso narrativo creado o consumido. "La narración transmite a través de sus personajes, espacios, tiempos y acciones, contenidos éticos que nos sitúan en el ámbito de los valores humanos. La estructura narrativa, por su naturaleza semio-narrativa, es capaz de transmitir la vida real o imaginaria de tal forma que sea comprendida fácilmente, y los sujetos receptores trasladen su empatía con los personajes de la película a las relaciones con las personas de su entorno próximo o lejano" (García García, 2011: 13). Cuestiones técnicas de aplicación inmediata son, por ejemplo, el formato del guión, la estructura, el conflicto, el subtexto, los diálogos, la especificidad de la escritura para cada medio..., 
mientras que cuestiones más generales son la narrativa y el estilo, los géneros, el documental.

E1 78\% de las ofertas analizadas mencionaban profusamente asuntos relacionados con la narrativa general y el estilo y el 70\% le dedicaba cierta atención al estudio de los géneros, pero a la vez que el $70 \%$ se esforzaba por explicar a los alumnos el guión desde sus rudimentos más elementales, la explicación del formato, las didascalias, etc... .

\begin{tabular}{|c|c|c|c|c|}
\hline  & SI & NO & $\mathrm{NS} / \mathrm{NC}$ & Total \\
\hline Formato de guión & $70 \%(16)$ & $22 \%(5)$ & $9 \%(2)$ & (23) \\
\hline Estructura & $74 \%(17)$ & $17 \%(4)$ & $9 \%(2)$ & (23) \\
\hline Personajes & $65 \%(15)$ & $26 \%(6)$ & $9 \%(2)$ & (23) \\
\hline El espacio & $26 \%(6)$ & $65 \%(15)$ & $9 \%(2)$ & (23) \\
\hline Nuevos soportes audiovisuales & $35 \%(8)$ & $61 \%(14)$ & $4 \%(1)$ & (23) \\
\hline Elaboración y presentación de proyectos & $70 \%(16)$ & $22 \%(5)$ & $9 \%(2)$ & (23) \\
\hline Géneros & $70 \%(16)$ & $22 \%(5)$ & $9 \%(2)$ & (23) \\
\hline El conflicto & $39 \%(9)$ & $52 \%(12)$ & $9 \%(2)$ & (23) \\
\hline Guión de cine & $87 \%(20)$ & $13 \%(3)$ & $0 \%(0)$ & (23) \\
\hline Guión de radio & $35 \%(8)$ & $65 \%(15)$ & $0 \%(0)$ & (23) \\
\hline Guión de cómic / historia gráfica & $9 \%(2)$ & $87 \%(20)$ & $4 \%(1)$ & (23) \\
\hline Guión de TV & $74 \%(17)$ & $26 \%(6)$ & $0 \%(0)$ & (23) \\
\hline Guión transmedia & $30 \%(7)$ & $65 \%(15)$ & $4 \%(1)$ & (23) \\
\hline El subtexto & $39 \%(9)$ & $52 \%(12)$ & $9 \%(2)$ & (23) \\
\hline El diálogo & $57 \%(13)$ & $35 \%(8)$ & $4 \%(1)$ & (22) \\
\hline Las adaptaciones & $35 \%(8)$ & $48 \%(11)$ & $17 \%(4)$ & (23) \\
\hline Los derechos y la autoría & $9 \%(2)$ & $83 \%(19)$ & $9 \%(2)$ & (23) \\
\hline Documentales & $52 \%(12)$ & $43 \%(10)$ & $4 \%(1)$ & (23) \\
\hline Falsos documentales & $43 \%(10)$ & $43 \%(10)$ & $13 \%(3)$ & (23) \\
\hline Narrativa y estilo & $78 \%(18)$ & $17 \%(4)$ & $4 \%(1)$ & (23) \\
\hline
\end{tabular}

Cuadro 1. Contenidos. Fuente: elaboración propia.

Un contenido intermedio entre lo que serían las técnicas de aplicación y los saberes más generales, nos parece que de vital importancia en tanto que afecta a lo humano más que cualquier otro, lo constituye el estudio del personaje. Como señalaba Egri (1960: 45) en los sesenta, "we understand the principle of change in character. A character is in constant change". Desde la Teoría del Guión siempre se intentaron fórmulas para enseñar/aprender a dibujar al ser humano a través de sus acciones a la vez que desde la Narrativa se buscaba entender el valor semántico de su comportamiento en el texto. Pudimos constatar una presencia significativa de este contenido en más de un $80 \%$ de casos, siendo extraordinariamente relevante en el $29 \%$.

Sin embargo y según puede observarse en el cuadro 2, sobre la intención de los contenidos, en muy pocos planes de estudio y guías docentes se observó que existiera un declarado deseo de utilizar la asignatura como vehículo para la formación de valores, tan solo un $18 \%$ lo declaraba de manera expresa. Sí, en cambio, se insistía bastante en 
que una de las misiones de la asignatura debía tener en cuenta la formación del criterio. Esto se podría deber en parte a que los conocimientos impartidos son tradicionalmente útiles no sólo para guionistas sino también para futuros productores ejecutivos, en tanto que capacitan no sólo para la escritura sino para desarrollar la capacidad valorativa al respecto de lo escrito.

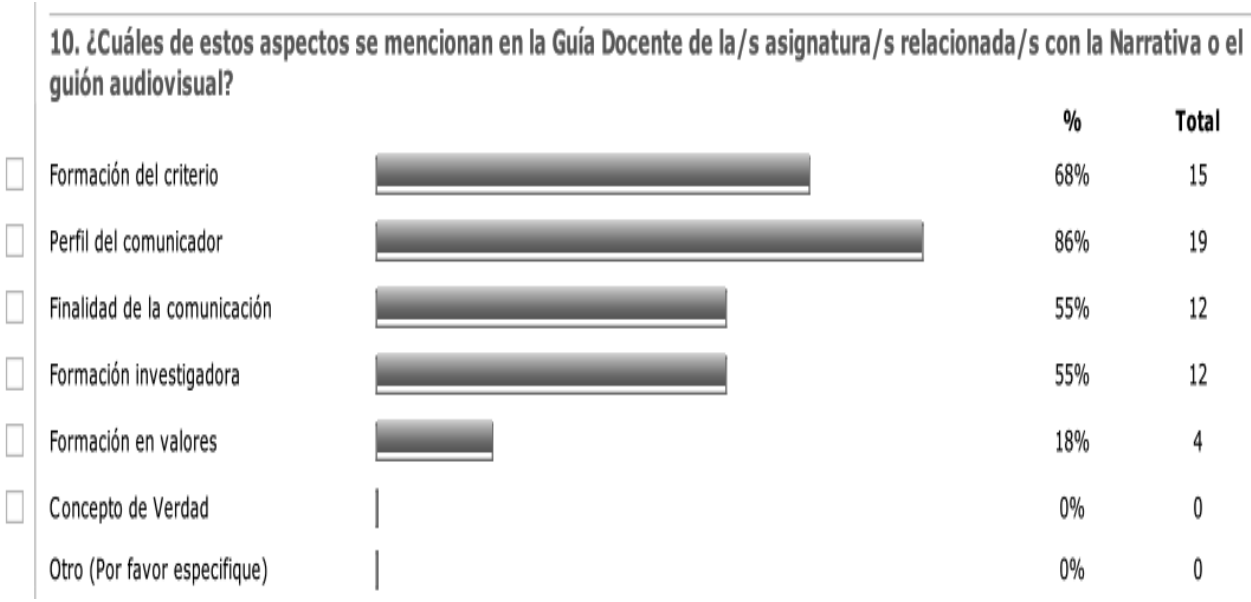

Cuadro 2. Intención Contenidos. Fuente: elaboración propia

Otros aspectos como el perfil del comunicador o la finalidad de la comunicación, encontraban reconocimiento en un importante número de casos, mientras que el papel de la asignatura en el marco de lo que sería un perfil investigador o, lo que sería casi lo mismo, la utilización de la asignatura para aprender a investigar se mencionaba en un $55 \%$ de casos. No es un porcentaje excesivo, si se tiene en cuenta por ejemplo, que la narratología sirve principalmente para ese cometido.

\subsection{Metodologías empleadas en las asignaturas}

En líneas generales la asignatura ha pasado de tener una consideración teórica a otra más eminentemente práctica. Las sesiones de teoría se redujeron a porcentajes que oscilaban entre el $25 \%$ y el $35 \%$. Aunque no hay datos ciertos, los que conocimos los primeros planes de estudios en este campo, sabemos que en sus orígenes la asignatura de Narrativa Audiovisual eran teóricos sin prácticamente ningún resquicio en el que el alumno pudiera poner en práctica lo aprendido y ser evaluado por ello.

Las diferencias ahora las encontramos sobre todo en el tipo de actividades prácticas que se propone a los alumnos. Éstas van desde visionados con coloquios posteriores o foros en plataformas virtuales, a ejercicios de escritura comentados que se realizan en ocasiones en laboratorios especializados, los cuales cuentan con programas específicos para la escritura de guiones, hasta la puesta en escena de lo escrito, la grabación y la posproducción del proyecto, esto último sólo en un 14\% de casos. 


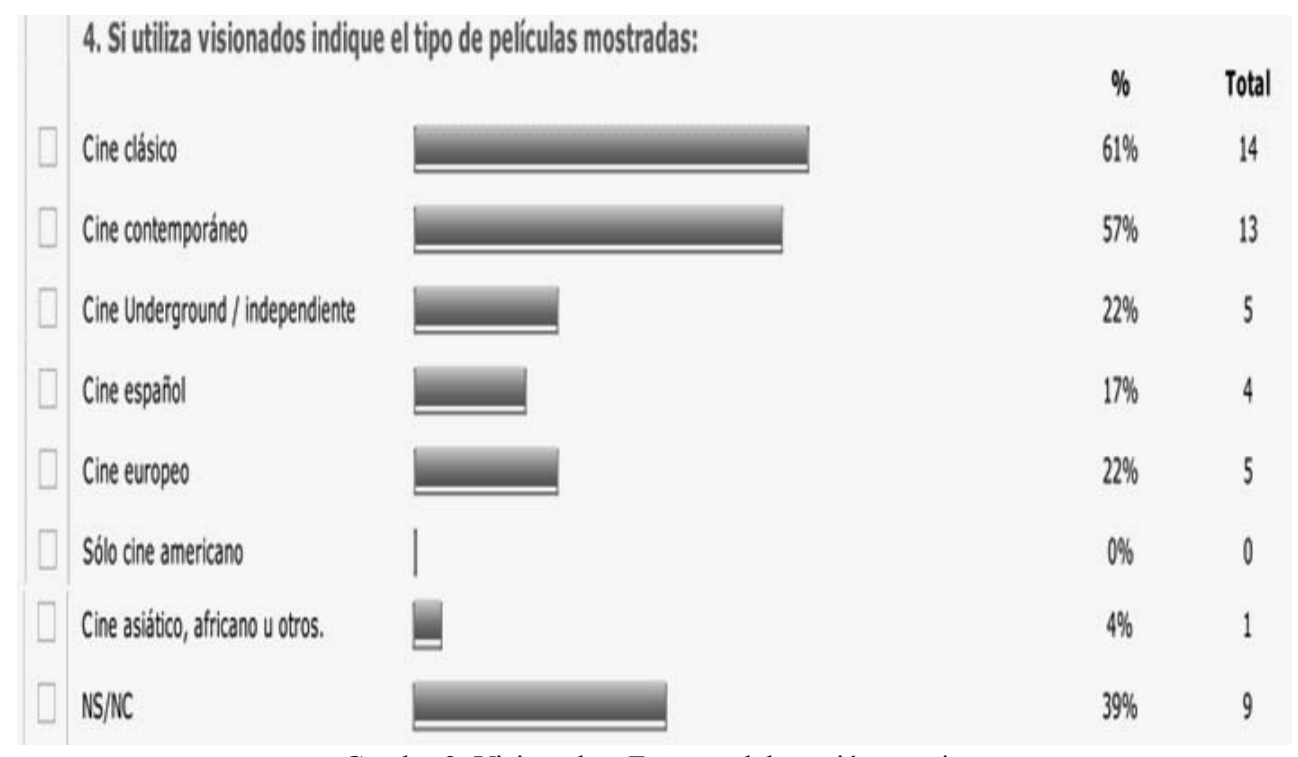

Cuadro 3. Visionados. Fuente: elaboración propia

Si observamos el cuadro 3, veremos un mapa aproximado de lo que se les muestra a los alumnos: cine clásico y contemporáneo se reparten la pantalla casi a partes iguales, mientras que por geografías, diríamos que el cine español y el europeo suponen, cada uno, la cuarta parte de lo mostrado a lo largo de un curso (de promedio) y que las cinematografías más marginales, como la africana o la asiática, apenas cuentan con representación por lo que cabe suponer que es el cine americano el más mostrado pero sin que ello suponga una mayoría abrumadora, recurrente o exclusiva, como quizá ocurriera en otras épocas.

Como hemos comentado, las prácticas también incluyen ejercicios de escritura y su comentario. Sorprende la ausencia de esta clase de prácticas en un $10 \%$ de las ofertas y que sean consideradas en la nota final en poco más de la mitad (55\%) lo que tal vez podría deberse a la dificultad que muchos docentes reconocen a la hora de valorar un trabajo creativo con objetividad, cuando tan a menudo se defiende que la subjetividad suele estar siempre detrás del juicio.

A este respecto nos cabe discrepar pues entendemos que lo que el docente debe valorar no es el resultado global como lo haría un crítico o un espectador, si no la pericia en la aplicación de las técnicas para la consecución de los objetivos que el propio alumno se haya marcado en el texto escrito o que se le hayan propuesto en el ejercicio.

En cuanto a las actividades complementarias relacionadas con la asignatura, se reconocían el análisis y la resolución de casos como las dos fórmulas más habitualmente ofrecidas.

Los alumnos intervienen en la ejecución de proyectos en un $67 \%$ de las titulaciones ofrecidas. Recordemos, sin embargo, que según datos anteriormente mostrados, el $82 \%$ no ejecutaba ni grababa los textos escritos, lo que resulta algo contradictorio. 
Cabe pensar aquí que el concepto de proyecto puede ser entendido en un sentido tan amplio como se quiera y que no se refiere exclusivamente a la obtención de un master final de vídeo.

\subsection{Fuentes empleadas en el ejercicio docente}

Las fuentes audiovisuales ya han sido grosso modo mencionadas, nos centraremos aquí en las fuentes bibliográficas. En este apartado se cuantificó qué autores aparecían en las bibliografías recomendadas, observándose un mayor impacto, como era de esperar, de ciertos autores sobre otros. Entre los autores más referenciados, destaca Robert Mckee con un mayor número de frecuencias.

Bastaría echar un vistazo para reconocer un triunfo de la pragmática sobre otras líneas más cercanas a la narratología, para asumir que la enseñanza de esta disciplina admite hoy poca demora frente al alumno, cada vez más partidario de soluciones prácticas y rápidas a los problemas planteados y mucho más reticente con los planteamientos teóricos de gran alcance. Parece claro que, cuando menos, ya se daba una tendencia en los últimos años a conciliar enfoques teóricos y prácticos, a superar las diferencias visibles también entre lo que el profesor García García define como la "escuela chomskiana y la pragmática norteamericana" (García García, 2006: 9).

Hay que tener en cuenta que los estudiantes llegan hoy a las universidades con una muy marcada prioridad que es la de conseguir conocimientos que les garanticen "una rentabilidad laboral de inmediata aplicación una vez finalizados los estudios" (García, 2011: 56). Aún así, no deja de ser sorprendente la incombustibilidad y recurrencia de clásicos como Aristóteles, en cuya poética como sabemos, están basadas la mayor parte de las obras preceptivas posteriores. Conseguir que un estudiante maneje la Poética no es tarea sencilla si no se refuerza la propuesta con algún mecanismo de control como podría ser un test de comprensión a través de la plataforma virtual o incluso de manera presencial, pero olvidarnos de introducirlo en las bibliografías supone una omisión, a nuestro juicio, poco justificable pues el alumno debe conocer cuáles son los orígenes del arte de la escritura, con independencia de que se anime a profundizar en ellos más o menos.

\section{Conclusiones}

La enseñanza de la Narrativa Audiovisual y de sus áreas más afines como la Teoría y Técnica del Guión Audiovisual, se ha reconvertido en los últimos años, hacia unas metodologías y unos contenidos teórico-prácticos mucho más basados en los modelos americanos, lo que sin duda puede entenderse como un giro hacia los aspectos más prácticos de la disciplina que la orientan indefectiblemente al ámbito de lo profesional.

Por otro lado y en relación con ello, los profesionales siguen desconfiando de los modelos docentes, defendiendo otros de naturaleza más bien autodidacta. La necesidad y la urgencia para encontrar soluciones específicas a problemas concretos es para ellos el elemento más motivador en el descubrimiento de teorías o recursos.

Desde un punto de vista didáctico, se podría pensar por tanto, que cualquier modelo de enseñanza-aprendizaje basado en la resolución de problemas o en la elabora- 
ción de proyectos, se situaría por este motivo de una manera clara, mucho más próximo a las necesidades formativas reales de guionistas, redactores y elaboradores de contenido en general.

Aunque el estudio nos muestra que tales metodologías se han implementado considerablemente, hay indicios para pensar que falta aún realizar un importante esfuerzo en este sentido, si consideramos que son pocas las ofertas que permiten la inmersión del alumno en todo el proceso que va desde la generación de una idea hasta la obtención del resultado final, tomando como base esta asignatura. Tal vez la mayoría de los proyectos se culminan desde otras áreas como la realización y la producción audiovisual pero esto, en nuestra opinión provoca el que cuando se abordan tales producciones se pueda descuidar el guión. Faltan experiencias integradoras. Una mayor vinculación entre la idea y su puesta en escena final, dentro de un mismo proyecto, mejoraría en nuestra opinión, la calidad de los resultados, tanto como del aprendizaje.

Entre los profesionales destaca una clara preocupación por aspectos tales como la adaptación a fórmulas de trabajo en grupo para las que muchas veces no se está preparado. La reconstrucción en el aula de estos ambientes, tal vez podría colaborar a un acercamiento de los alumnos a la realidad del guión y de la creación de los contenidos.

Entre los vacíos más visibles de la oferta académica descubrimos dos: por un lado la ausencia de especificidad de planteamientos teórico-prácticos en campos tales como los contenidos transmedia y la ausencia también de planteamientos éticos y vitales que elevarían considerablemente la capacidad crítica de los estudiantes.

Respecto a este segundo asunto, parece inevitable insistir en el papel de responsabilidad social de los medios y de quienes deben decidir sobre el qué y el cómo de los contenidos. Pueden, al hacerlo, orientar a la sociedad en direcciones mejores o peores. Lejos de sugerir un adoctrinamiento específico lo que planteamos aquí es la necesidad de que la asignatura sirva para que el alumno se haga preguntas que de algún modo nos interesan a todos y que, de esta manera, no desaproveche por falta de aptitud, cualquier situación que se le pueda presentar en su trayectoria profesional para contribuir de forma convincente a la consecución de un mundo más justo en el que los abusos sean denunciados sin temor y en el que los contenidos de ficción no se limiten a ser una mera escapada de la realidad que nos aleje de la obligación de intervenir en ella.

\section{Referencias bibliográficas}

EGRI, Lajos (1960): The art of dramatic writing. Its Basis in the Creative Interpretation of Human Motives. New York, Touchstone.

GARCÍA GARCÍA, Alberto (2010): "Espacio Europeo de Educación Superior”, en VIVAR ZURITA et al: Los estudios de comunicación en España: reflexiones en torno al Libro Blanco. Madrid, Icono 14, pp. 47-61.

GARCÍA GARCÍA, Francisco \& RAJAS, Mario (edit., 2011): Narrativas audiovisuales: el relato. Madrid, Icono 14.

GARCÍA GARCÍA, Francisco (2006): Narrativa Audiovisual. Madrid, Laberinto. 
JENKINS, Henry (2006): "Confronting the challenges of participatory culture: Media education for the 21st century", en Chicago: The John D. and Catherine McArthur Foundation. Disponible en: http://www.digitallearning.macfound.org/ [fecha de consulta: 21 de marzo de 2012].

LÓPEZ DE QUINTANA, Eugenio (2007): “Transición y tendencias de la documentación en televisión: digitalización y nuevo mercado audiovisual", en El profesional de la información, Vol. 16, ${ }^{\circ} 5$. Disponible en:

http://elprofesionaldelainformacion.metapress.com/app/home/contribution.asp?referrer $=$ parent $\&$ backto $=$ issue $, 1,18 ;$ journal, 30,84; linkingpublicationresults,1:105302,1 [fecha de consulta: 5 de julio de 2012].

MCKEE, Robert (1997): Story. Substance, structure, style, and the principles of screenwriting. New York City, Harper Collins Publishers.

RIOS CARRATALÁ, Juan Antonio (2006): “Los peligros del guión”, en Edición digital a partir de las relaciones entre el cine y la literatura: el guión. Alicante, Universidad de Alicante, 1997, 99 11-20. Disponible en:

http://www.cervantesvirtual.com/obra/los-peligros-del-guin-0/ [fecha de consulta: 22 de agosto de 2012].

ROOT-BERNSTEIN, Robert \& ROOT-BERNSTEIN, Michèle (2002): El secreto de la creatividad. Barcelona, Kairós.

\section{Dr. Pedro Javier GÓMEZ MARTÍNEZ}

Universidad Francisco de Vitoria

Profesor de Comunicación

Guionista de televisión

p.gomez.prof@ufv.es

\section{Dr. Miguel Ángel POVEDA CRIADO}

Universidad Francisco de Vitoria

Profesor de Comunicación

Productor audiovisual

m.poveda.prof@ufv.es 